\title{
Comparison between an immunochemical fecal occult blood test and a Guaiac based fecal occult blood test in detection of adenomas and colorectal cancer
}

\author{
Bruna Elisa Catin Kupper ${ }^{1 *}$, Samuel Aguiar Juniorr', Wilson Toshihiko Nakagawa', Renata Mayumi Takahashi ${ }^{1}$, \\ Ranyell Matheus Spencer Sobreira Batista', Tiago Santoro Bezerra', Paulo Roberto Stevanato Filho', \\ Thiago Celestino Chulam $^{2}$ and Ademar Lopes ${ }^{1}$
}

\begin{abstract}
Background: Colorectal cancer (CRC) is the third most commonly diagnosed cancer worldwide. Brazil does not have an official well established program for screening colorectal cancer. The aim of this study was to compare Guaiac Based Fecal Occult Blood Test (G-FOBT) to a kind of an Immunochemical Fecal Occult Blood Test (I-FOBT), in search of cancer or advanced adenoma.

Methods: Prospective and cross-sectional study. Asymptomatic and average-risk individuals $(n=1500)$ aged from 50 to 75 years old were invited to participate in the study. The primary endpoint was positivity rate and the secondary endpoints were adherence rate and significant endoscopic findings. All participants received both tests with follow-up colonoscopy if either test was positive.

Results: Adherence rate of G- FOBT was 756/1500 (50.4\%) while for I- FOBT it was 960/1500(64\%). The positivity ratio in the I- FOBT was 94/960 (9.8\%) and in the G-FOBT was 20/771 (2.6\%). The Positive Predict Value (PPV) for the I- FOBT counted 16/77 (21.0\%) while for the G- FOBT it was 6/18 (33.0\%), considering significant lesions. Regarding the colorectal cancer findings, the detection in the colonoscopy guided from the positivity of fecal occult blood tests was 5/77 (6.5\%) in I- FOBT and 2/18 (11.1\%) on the G- FOBT.

Conclusions: The positivity, the adherence rate and the capacity to detect significant lesions were higher in I-FOBT. Considering the findings of the study we could conclude that I-FOBT was superior to G- FOBT.

Trial registration: This study was reviewed and approved by the Institutional Review Board of A.C.Camargo Cancer Center, São Paulo, Brazil, number: 1877/14.
\end{abstract}

Keywords: Colorectal Neoplasms, Mass screening, Early detection of cancer

\section{Background}

Colorectal cancer (CRC) is the third most commonly diagnosed cancer with nearly 1.4 million cases diagnosed and 694,000 deaths worldwide in 2012. It is predicted that by 2035, 2.4 million cases will be diagnosed every year. More than a half of all the cases are from developed region. The most incident regions are Australia,

\footnotetext{
* Correspondence: bruna.sousa@accamargo.org.br

${ }^{1}$ Colorectal Cancer Department, A.C.Camargo Cancer Center, Adress: Taguá,

440, São Paulo CEP: 01508-010, Brazil

Full list of author information is available at the end of the article
}

Europe and North America, were the incidence are more than 30 new cases per 100.000 individual per year [1].

In Brazil colorectal cancer is also the third most incident neoplasia. 32,600 new cases were estimated for 2014. The disease distribution is heterogeneous due to socioeconomic differences among the regions of Brazil, being more incident in the ones which higher levels of development and population density [2].

Survival in CRC patients is primarily related to the stage of cancer at the time of diagnosis. The 5-year 
survival is achieved for $\geq 95 \%$ for early-stage disease, but for metastatic disease it is less than $\leq 10 \%$ [3].

$\mathrm{CRC}$ is believed to develop from non-malignant precursor adenomas. Progression from an adenoma to CRC is thought to take about 10 years and detection and removal of adenomas or early stage cancers reduce mortality from CRC [4]. Malignant cancer has a preclinical asymptomatic stage, and it is often not until the cancer has progressed to advanced cancer that symptoms occur.

According to the CDC (Centers for Disease Control, USA), it will be possible to achieve $60 \%$ reduction in deaths from CRC if all individuals older than 50 years are screened [5].

Despite the high incidence and mortality of colorectal cancer justify the search for effective prevention strategies, Brazil lacks a national population- based screening for CRC. A population-based screening program requires invites the entire target population, according to explicit and pre-defined protocols, with a testing method that is convenient enough for the target population to undertake and one that is affordable to the central funding organization [6].

The United States Prevention Service (US-PSTF) recommends the screening for CRC of asymptomatic individuals between 50 and 75 years old through the following detection methods: 1) Immunochemical Fecal Occult Blood Test (I-FOBT) held annually with colonoscopy in case of the positivity of occult blood test; 2) IFOBT held every 3 years with sigmoidoscopy every 5 years and colonoscopy in case of the positivity of occult blood test; 3) colonoscopy every 10 years [7].

Colonoscopy is the 'gold standard' method for the colorectal cancer diagnostic and prevention, not only because of its high sensibility but also because of the capacity of removing adenomas. Very recent studies showed reduction on mortality and incidence taxes of colorectal cancer in population screened for it and submitted to polypectomy when adenomas were detected [6-9].

In spite of all benefits of this exam, colonoscopy is an invasive procedure and requires hospital attendance and administration of a bowel preparation that is also inconvenient and unpleasant for the patient. The need for highly skilled practitioners, using expensive equipment and facilities, and stringent assurance procedures makes colonoscopy an expensive option for population screening. For this reason, it is usually necessary to employ a primary screening test like fecal occult blood test, that can identify people at greatest risk of CRC for whom invasive tests, as colonoscopy, is appropriated [10-12].

Adenomas and cancerous lesions in the colon can bleed and this can be detected in stool by fecal occult blood tests. There are two popular analytical techniques to measure hemoglobin $(\mathrm{Hb})$ in feces. The Guaiac-based Fecal Occult Blood Test (G-FOBT) that detects the heme component of $\mathrm{Hb}$, and the Immunochemical Fecal Occult Blood Test (I-FOBT) that detects the globin component of $\mathrm{Hb}[6]$.

Despite the existence of global studies comparing immunochemical method and the Guaiac method in the detection of CRC and advanced adenomas, the only FOBT available in the Brazilian public health system is the Guaiac Test and studies in Brazilian based cases comparing adherence and performance between the two tests are limited.

Studies have shown that adherence to the FOBT and colonoscopy vary according not only to ethnic and sociodemographic characteristics, but also to personal health beliefs, which highlight the importance of studying the population to promote appropriate campaigns to each population profile $[6,13,14]$.

Given the importance of this subject and the knowledge gap regarding the adherence of the Brazilian population to the different screening methods available for CRC, this study aimed to compare the adherence and the positivity value between two different tests of fecal occult blood (I- FOBT and G-FOBT) in the detection of significant colorectal neoplasia (advanced adenomas or colorectal cancer) in a population of asymptomatic individuals and at intermediate risk for colorectal cancer.

\section{Methods}

This is a cross-sectional study, with prospective data collection. In 2014, between April and July, 1500 asymptomatic and average-risk individuals aged between 50 and 75 were invited to participate in this pilot early colorectal cancer screening program. This study was reviewed and approved by the Institutional Review Board of A.C.Camargo Cancer Center, São Paulo, Brazil, number: 1877/14. All individuals, recruited to participate voluntarily in the study, were participating in a screening program for different kinds of neoplasia in a hospital situated in Sao Paulo, Brazil and signed the written informed consent for the study. Individuals should be residents of Sao Paulo metropolitan region and not have any private health insurance. The personal history of CRC/polyps or the diagnosis of hereditary colorectal cancer syndrome was an exclusion criterion for the enrollment. The subjects assigned a consent informed term and received oral and written instructions about how to collect the stool samples, as well as the two kinds of test tubs to collect feces for the G-FOBT and I-FOBT. They were informed that it would be important to have both tests. G-FOBT and I-FOBT were processed independently and participants were asked to take their completed test to the same hospital that they were recruited to the study. The return of the stool sample using a mailing service was not an option, therefore the participants should go by themselves to the lab. 
The I-FOBT used in this study was from a qualitative type (OC-Light: Eiken Chemical Co. Ltd., Tokyo Japan). For this test no diet or drug restriction was required. Each participant was provided with a test tube of the I-FOBT and instructed to take a stool sample. For the I-FOBT the test sampling device is shaped like a small test tube with the fecal probe inserted into it and sealing it. The probe has a serrated tip which is poked into four different areas of the stool and then pushed back into the tube. The probe tip with the fecal sample is immersed in a standard volume of hemoglobin stabilizing buffer. The presence of a globin antibody complex was identified as a visible line that reveals the presence of globin in the sample when levels are as low as $50 \mathrm{ng} / \mathrm{ml}$. The positive result is expected within $5 \mathrm{~min}$. A second reader independently interpreted the result to confirm it. In cases of discordance in the interpretation of the test, the technicians agreed on the positivity or negativity of the test after discussion.

To collect the stool sample for the G-FOBT, participants were asked to abstain from meat, some vegetables, fruits containing vitamin $\mathrm{C}$, alcoholic drinks and anticoagulant drugs. Participants were responsible to collect three stool samples in a dry tube and return this to lab. They were also asked to store their stool sample in the refrigerator if they were unable to return this immediately after they had completed it, and were informed that the validity of this test was $24 \mathrm{~h}$ if refrigerated or two hours in ambient temperature. The lab staffs were responsible to check if participants complied with all the instructions concerning stool collect and refrigeration. If there were any kind of mistake concerning the collect or storage, the stool were annul and the patient were asked to collect another one.

The G-FOBT is a chemical method. In the presence of the heme component of hemoglobin, oxygen released from hydrogen peroxidase oxidize guaiac, a phenolic compound present in the resin of a South American hardwood tree (Guaiacum officinale), to form a bluecoloured compound $[6,15,16]$. In the lab, the analyst had to smear a small sample of the feces onto the test card treated with guaiac resin and adds hydrogen peroxide to the sample. The test is positive if a blue colour appears on the card paper within $60 \mathrm{~s}$.

If either of the tests were positive, the subject was informed about the result by a phone call and a colonoscopy was booked as part of the study. Colonoscopy was recommended if at least one test was positive. The primary endpoint was positivity rate and the secondary endpoints were adherence rate and significant endoscopic findings.

Qualified gastroenterologists performed the colonoscopies. They were blinded as to which test(s) proved positive. Pathologists provided in their report the number of adenomas, their size, location, histology and dysplasia grade. If a patient had more than one adenoma, the most advanced lesion was included in the study analysis. Advanced adenoma was defined as an adenoma of $10 \mathrm{~mm}$ or more in size, or with high-grade dysplasia or a villous component. Cancers were classified according to the TNM classification. The significant lesions were described as advanced adenoma or cancer [17].

\section{Statistical analysis}

The patient's most severe pathologic finding was recorded, concerning histological characteristics, polyp size, and number of polyps. For categorical data we analyzed frequencies. Both test results were analyzed independently and colorectal cancer and advanced adenoma were defined as clinically significant colorectal neoplasia.

The adherence rate of I-FOBT and G-FOBT were compared by using the chi-square test. For all tests we adopted 0.05 significance level. We also reported 95\% Confidence Intervals (CI) for these differences in the detection rates between the two tests. Mean, minimum and maximum values were decrypted for quantitative data. To compare proportions between groups were used hypothesis test.

Statistical analyses were performed using $\mathrm{R}$ version 2.15.1 (R Foundation for Statistical Computing, Vienna, Austria) and SPSS for Windows software, version 20.0 (SPSS Inc., Chicago, Illinois).

Since colonoscopy was restricted to subjects classified as positive by at least one of the tests, the sensitivity and specificity of each test could not be estimated directly.

\section{Results}

Characteristics of the study population are shown in Table 1. From the individuals recruited, 420 (28\%) were men and $1080(72 \%)$ were women and the average age was $59(50-75)$ years old. The adherence rate was significantly different $(p<0.001)$ for G-FOBT $756(50.4 \%)$ and IFOBT 960 (64\%). The positivity ratio for I-FBOT was 94 (9.8\%) and for the G-FOBT it was 20 (2.6\%) (Table 2).

Concerning the colonoscopies, 77 (81.9\%) of I-FOBT positive patients underwent this exam while for the

Table 1 Clinical characteristics of individuals recruited for the study

\begin{tabular}{|c|c|c|c|c|}
\hline Participant characteristics & $n$ & & $\%$ & \\
\hline Enrolled & 1500 & & - & \\
\hline \multicolumn{5}{|l|}{ Sex } \\
\hline Male & 420 & & 28 & \\
\hline Female & 1080 & & 72 & \\
\hline \multicolumn{5}{|l|}{ Age } \\
\hline & Min & Mean & Max & Std Dev \\
\hline & 50 & 59.9 . & 75 & 6.5 \\
\hline
\end{tabular}


Table 2 Comparision of adherence

\begin{tabular}{lllll}
\hline & G-FOBT (\%) & I-FOBT (\%) & $p$ & IC (95\%) \\
\hline Adherence rate & $756(50.4)$ & $960(64.0)$ & $p<0.0001$ & {$[0.09 ; 0.16]$} \\
Positivity rate & $20(2.6)$ & $94(9.8)$ & & \\
$\begin{array}{l}\text { Colonoscopies } \\
\text { Performed }\end{array}$ & $18(90)$ & $77(81.9)$ & & \\
$\begin{array}{l}\text { Significant } \\
\text { Colonocopy findings }\end{array}$ & $6 / 18(33.0)$ & $16 / 77(21.0)$ & $p=0.26$ & {$[-0.09 ; 0.34]$} \\
\hline
\end{tabular}

G-FOBT $18(85.0 \%)$ of the patient attended it. Among the colonoscopy findings, individuals that had the IFOBT positive numbered $20(26.0 \%)$ non-advanced adenomas, 11 (14.3\%) advanced adenomas and 5 (6.5\%) invasive colorectal cancer, whereas in G-FOBT positive group, colonoscopies evidenced 4 (22.2\%) non-advanced adenomas, 4 (22.2\%) advanced adenomas and 2 (11.1\%) invasive colorectal cancer.

About the adenomas characteristics, most of them were tubular, sized between 6 and $9 \mathrm{~mm}$, and most individuals had more than three adenomas in the colonoscopies (Table 3). Most of invasive carcinomas were stage 1 for the I- FOBT (80.0\%) and half of them were stage 1 for G-FOBT. The number of colonoscopies required to detect one invasive or non-invasive CRC was 15.4 with I-FOBT and 9.0 with G-FOBT (Table 3). The Positive Predict Value (PPV) for the I- FOBT counted 16/77 (21.0\%) while for the G- FOBT it was 6/18 (33.0\%), considering significant lesions.

\section{Discussion}

The main finding of this study was a higher rate of advanced adenomas and cancer, defined as significant lesions, detected using the I-FOBT than G-FOBT. The adherence to the I-FOBT was also statically higher than the adherence to the G-FOBT in this study.

The design of the I-FOBT and the need of only one sample are probably the main reason for the higher adherence to this method. A meta-analysis publicized in 2012, evaluated randomized control trials comparing guaiac fecal occult blood test with fecal immunochemical test participation rates. According to this meta- analysis the implementation of I-FOBT is likely to increase participation in CRC screening. Four of the studies of this review concluded that participation rates in the IFOBT group were higher because they do not have dietary or medicinal restrictions [18].

The low cost and simple analysis have enhanced the utility of G-FOBT for many population-based screening programs however the disadvantages associated with GFOBT are not only limited by the number of stool samples that is required. This test is also more leaning to false-positive or false-negative results according to the diet. The consumption of Antioxidants such as ascorbic
Table 3 Most advanced lesions Identified by screening

\begin{tabular}{|c|c|c|}
\hline & G-FOBT (\%) & I-FOBT (\%) \\
\hline \multicolumn{3}{|l|}{ Colonoscopies Findings } \\
\hline Normal & $4(22.2)$ & $19(24.6)$ \\
\hline Benign lesions & $4(22.2)$ & $22(28.6)$ \\
\hline Nonadvanced adenomas & $4(22.2)$ & $20(26.0)$ \\
\hline Advanced adenomas & $4(22.2)$ & $11(14.3)$ \\
\hline Invasive Colorectal Cancer & $2(11.1)$ & $5(6.5)$ \\
\hline Total & $18(100.0)$ & $77(100.0)$ \\
\hline \multicolumn{3}{|l|}{ Adenomas characteristics } \\
\hline \multicolumn{3}{|l|}{ Histology } \\
\hline LGD: Tubular & $5(62.5)$ & $24(77.4)$ \\
\hline LGD: Tubulovillous or villous & $3(37.5)$ & $7(22.6)$ \\
\hline Total & $8(100.0)$ & $31(100.0)$ \\
\hline \multicolumn{3}{|l|}{ Size } \\
\hline$\leq 5 \mathrm{~mm}$ & $2(25.0)$ & $7(22.6)$ \\
\hline $6-9$ mm & $4(50.0)$ & $13(41.9)$ \\
\hline$\geq 10 \mathrm{~mm}$ & $2(25.0)$ & $11(35.5)$ \\
\hline Total & $8(100.0)$ & $31(100.0)$ \\
\hline \multicolumn{3}{|l|}{ Number } \\
\hline$<3$ & $7(87.5)$ & $22(70.9)$ \\
\hline$\geq 3$ & $1(12.5)$ & $9(29.1)$ \\
\hline Total & $8(100.0)$ & $31(100.0)$ \\
\hline \multicolumn{3}{|l|}{ Invasive carcinoma stages } \\
\hline Stage 1 & $1(50.0)$ & $4(80.0)$ \\
\hline Stage 2 & $1(50.0)$ & $1(20.0)$ \\
\hline Stage 3 & 0 & 0 \\
\hline Total & $2(100.0)$ & $5(100.0)$ \\
\hline
\end{tabular}

acid inhibit the oxidation of guaiac and have the potential of causing false- negative results, furthermore GFOBT is not specific to the human hemoglobin, so the presence of meat intake, has the potential to cause a false- positive test result. Moreover, the heme group of human hemoglobin is very resistant even when subjected to the digestive process in the gut. For this reason blood from the upper gastrointestinal tract can sometimes be detected in stool [15, 19-21].

The higher positivity rate of the I-FOBT compared with the G-FOBT is related to the lower detection level of occult blood, making I-FOBT less specific and more sensitive than G-FOBT [20]. As the G-FOBT is not really sensitive for blood detection, this test can under detect some initial CRC and advanced adenomas that are not bleeding significantly.

A study conducted in Florence, Italy, and publicized in 2015 , analyzed the risk of developing CRC in the 11 years following an initial I-FOBT (carried out in the mid1990s), and compare it with subjects who, although 
invited, did not undergo the test during the same period of time. The study conclusion was that the implementation of CRC screening based on a single I-FOBT every 2 years, reduces CRC incidence even with only a few screening rounds. This study also observed a reduction in CRC mortality of $41 \%$ for the adherents as compared to the non-adherents for the I-FOBT. They also emphasized that, in spite of an initially increase in CRC incidence in the first years of screening, due to the early diagnosis of prevalent CRCs, after a period of about 6-7 years of screening a dramatic decrease in CRC incidence for the attendees was observed [22].

In contrast, another recent study, that also aimed to determine the effect of CRC screening using a guaiac FOBT, concerning on CRC mortality and incidence, was conducted in France. This cohort population-based study with average risk individuals over a period of 22 years, demonstrated no effect on cancer incidence but significantly reduced CRC mortality by $13 \%$, after 11 screening rounds. This major finding suggests that the proportion of advanced adenomas identified by guaiac FOBT, due to their poor sensitivity, is too small to cause a reduction in CRC incidence [23].

The detection of cancer was at least twice as high with the I-FOBT as with the G-FOBT, and the number of advanced adenomas was about two to three times as high. Similar results have been reported in other populationbased studies around the word. [19, 24]. It is reasonable to presume that a national population-based screening program using an I-FOBT would lead to a greater reduction in mortality. On the other hand the increased number of colonoscopies could lead to problems of colonoscopy capacity, especially in Brazil.

An Italian prospective study showed that CRC patients diagnosed through screening had increased 5 years on cancer-related survival rate when compared with symptomatic patients. This survival benefit appears to be related entirely to the ability of I-FOBT screening to detect a higher proportion of cancers at an earlier stage than is seen in symptomatic patients, as there is no difference in anatomical location of tumors or in the age at diagnosis between screen-detected and pre-screening cohorts $[25,26]$.

Some design limitations of this study may have had an important impact on the participation rate and adherence to the different tests. As the study population had voluntarily already consented to participate in a screening program, the adherence to both types of FOBTs was maybe higher than it would have been if the tests were adopted in a national screening program once in the study scenario the population had a previous wish of being exposed to screening exams.

Another important point that maybe had a negative impact on the adherence to GFOBT was the method adopted to collect stool for this exam. The participants had to fill stool containers instead of completing guaiac cards. Despite the fact that most centers around the world collect the stool sample in the guaiac card, the collection of stool in a dry tube is a method adopted by different labs that use G-FOBT in Brazil and was the method adopted as a standard procedure in this screening program. The necessity of maintaining the stool samples refrigerated, the limited time to return the sample to the lab and the absence of a mail service is possibly negative points to the participation rate in the G-FOBT, however, it reflects the real scenario of this screening campaign and the reality of many diagnosis centers in Brazil.

It is important to highlight that all the participants that had a positive result in any of the FOBTs were invited to perform the colonoscopy but only $90 \%$ of the patients that had a positive result in the IFOBT and $81.9 \%$ of the patients that had a positive result in the G-FOBT were followed up with colonoscopy because they refused to do the exam or didn't answer the phone or mail contact.

lso the I-FOBT and G-FOBT used in this study were qualitative tests. Therefore, reading and interpreting the test is subjective since it depends on the analyses of the reader. Once the cutoff values cannot be adjusted, the result.

provided in the two tests is only positive or negative what seems to act as a limiting factor, because in this kind of analyses it is impossible to know exactly how much blood is present in the stool sample.

The use of a quantitative and automated I-FOBT would improve precision, increase analysis through eliminating the subjective nature associated with both G-FOBT and the qualitative I-FOBT used. Quantitative I- FOBT is being used in population-based screening programs in many countries including Australia, Japan, Italy, and Canada. Other studies to investigate the usage of this kind of exam in Brazil, as well as other studies to evaluate the cost effectiveness of colorectal cancer screening in Brazil, would afford knowledge to the adoption of a national population-based screening program in the future.

\section{Conclusions}

The positivity, the adherence rate and the capacity to detect significant lesions were higher in I-FOBT. Considering the findings of this study we could conclude that I-FOBT was superior to G-FOBT in the screening of this asymptomatic population. Given the superior performance characteristics of the I-FOBT, it is seems to be clear that an national organized program using this type of test would lead to reduction in CRC mortality and a possible effect on CRC incidence, although more studies concerning colorectal cancer screening in Brazil should be conducted with the objective to investigate invitation strategies to improve fecal occult blood test participation. 


\section{Abbreviations}

CCR: Colorectal cancer; CDC: Centers for Disease Control- USA; GFOBT: Guaiac based fecal occult blood test; I-FOBT: Immunochemical fecal occult blood test; US-PSTF: United States prevention service

\section{Acknowledgments}

The authors thank the patients for their cooperation and Eiken, Fujifilm, and Japanese Government for the grants.

\section{Funding}

This study was partially supported by Eiken, Fujifilm, and Japanese Government.

\section{Availability of data and materials}

Please contact author for data requests.

\section{Authors' contributions}

SAJ, WTN and AL conceived of the study. BECK and SAJ participated in the design of the study, performed the statistical analysis, coordinated the study and helped to draft the manuscript. All authors participated in the data collection, in its design, statistical analysis, read and approved the final manuscript.

\section{Ethics approval and consent to participate}

This study was reviewed and approved by the Institutional Review Board of A.C.Camargo Cancer Center, São Paulo, Brazil, number: 1877/14. Writen consent to participate in the study was taken from all patients included in the study. The study was performed in complete compliance with the Helsinki Declaration.

\section{Consent for publication}

Not applicable.

\section{Competing interests}

Authors declare that this study was partially supported by Eiken Chemical, Fujifilm, and Japanese government.

\section{Publisher's Note}

Springer Nature remains neutral with regard to jurisdictional claims in published maps and institutional affiliations.

\section{Author details}

${ }^{1}$ Colorectal Cancer Department, A.C.Camargo Cancer Center, Adress: Taguá, 440, São Paulo CEP: 01508-010, Brazil. ²A.C.Camargo Cancer Center, São Paulo, Brazil.

\section{Received: 8 November 2017 Accepted: 17 January 2018}

Published online: 15 February 2018

\section{References}

1. Ferlay J, Soerjomataram I, Ervi M, Dikshit R, Eser S, Mathers C, et al. Cancer incidence and mortality worldwide: IARC CancerBase No. 11 [Internet]. GLOBOCAN 2012 v1.0. Lyon: International Agency for Research on Cancer; 2013. Available from: http://globocan.iarc.fr

2. INSTITUTO NACIONAL DE CÂNCER (Brasil). Estimativa 2014: Incidência de Câncer no Brasil. Rio de Janeiro: INCA; 2014. Available from: http://WwW. saude.sp.gov.br/resources/ses/perfil/gestor/homepage/outros-destaques/ estimativa-de-incidencia-de-cancer-2014/estimativa_cancer_24042014.pdf

3. De Angelis R, Sant M, Coleman MP, et al. EUROCARE-5 Working Group. Cancersurvival in Europe 1999-2007 by country and age: results of EUROCARE-5-apopulation-based study. Lancet Oncology. 2014;15:23-4.

4. Winawer SJ, Fletcher RH, Miller L, Godlee F, Stolar MH, Mulrow CD, et al. Colorectal cancer screening: clinical guidelines and rationale. Gastroenterology. 1997;112(2):594-642.

5. Centers for Disease Control. (2014). Colorectal cancer screening:It's the right choice infographic. Atlanta: Centers for Disease Control, 2014. Disponível em http://www.cdc.gov/cancer/colorectal/basic_info/screening/infographic. htm. Accessed 07 Fed 2015.

6. Benton SC, Seaman HE, Halloran SP. Faecal occult blood testing for colorectal cancer screening: the past or the future. Curr Gastroenterol Rep. 2015:17(2):428

7. U.S. Preventive Services Task Force. Screening for colorectal cancer: U.S. Preventive Services Task Force recommendation statement. Ann Intern Med. 2008;149(9):627-37.
8. Zauber AG, Winawer SJ, O'Brien MJ, Lansdorp-Vogelaar I, van Ballegooijen M, Hankey BF, et al. Colonoscopic polypectomy and long-term prevention of colorectal-cancer deaths. N Engl J Med. 2012;366(8):687-96.

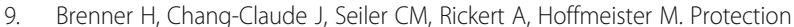
from colorectal cancer after colonoscopy: a population-based, case-control study. Ann Intern Med. 2011;154:22-30.

10. Panteris V, Haringsma J, Kuipers EJ. Colonoscopy perforation rate, mechanisms and outcome: from diagnostic to therapeutic colonoscopy. Endoscopy. 2009;41(11):941-51.

11. Kaminski MF, Bretthauer M, Zauber AG, Kuipers EJ, Adami HO, van Ballegooijen $M$, et al. The NordICC Study: rationale and design of a randomized trial on colonoscopy screening for colorectal cancer. Endoscopy. 2012;44(7):695-702.

12. Lin OS. Performing colonoscopy in elderly and very elderly patients: Risks, costs and benefits. World J Gastrointest Endosc. 2014;6(6):220-6.

13. Walsh JM, Kaplan CP, Nguyen B, et al. Barriers to colorectal cancer screening in Latino and Vietnamese Americans. Compared with non-Latino white Americans. J Gen Intern Med. 2004;19(2):156-66.

14. Ojinnaka C, Vuong A, Helduser J, Nash P, Ory MG, McClellan DA, Bolin JN. Determinants of variations in self-reported barriers to colonoscopy among uninsured patients in a primary care setting. J Community Health. 2015; 40(2):260-70.

15. Greegor $\mathrm{DH}$. Occult blood testing for detection of asymptomatic colon cancer. Cancer. 1971;28(1):131-4.

16. Greegor DH. Diagnosis of large-bowel cancer in the asymptomatic patient. JAMA. 1967:201:943-5.

17. Winawer SJ, Zauber AG. The advanced adenoma as the primary target of screening. Gastrointest Endosc Clin N Am. 2002;12(1):1-9.

18. Vart G, Banzi R, Minozzi S. Comparing participation rates between immunochemical and guaiac faecal occult blood tests: a systematic review and meta-analysis. Prev Med. 2012;55(2):87-92

19. Van Rossum LG, van Rijn AF, Laheij RJ, Van Oijen MG, Fockens P, van Krieken $\mathrm{HH}$, et al. Random comparison of guaiac and immunochemical fecal occult blood tests for colorectal cancer in a screening population. Gastroenterology. 2008;135(1):82-90.

20. Kuipers EJ, Rosch T, Bretthauer M. Colorectal cancer screening-optimizing current strategies and new directions. Nat Rev Clin Oncol. 2013:10(3):130-42.

21. Duffy MJ, Lamerz R, Haglund C, Nicolini A, Kalousová M, Holubec L, et al. Tumor markers in colorectal cancer, gastric cancer and gastrointestinal stromal cancers: European group on tumor markers 2014 guidelines update. Int J Cancer. 2014;134(11):2513-22.

22. Ventura L, Mantellini P, Grazzini G, Castiglione G, Buzzoni C, Rubeca T, Sacchettini C, Paci E, Zappa M. The impact of immunochemical faecal occult blood testing on colorectal cancer incidence. Dig Liver Dis. 2014; 46(1):82-6.

23. Hamza S, Cottet V, Touillon N, Dancourt V, Bonithon-Kopp C, Lepage C, Faivre J. Long-term effect of faecal occult blood screening on incidence and mortality from colorectal cancer. Dig Liver Dis. 2014;46(12):1121-5.

24. Park DI, Ryu S, Kim YH, et al. Comparison of guaiac-based and quantitative immunochemical fecal occult blood testing in a population at average risk undergoing colorectal cancer screening. Am J Gastroenterol. 2010;105:2017-25.

25 Faivre J, Dancourt V, Denis B, Dorval E, Piette C, Perrin P, Bidan JM, Jard C, Jung $S$, Levillain R, Viguier J, Bretagne JF. Comparison between a guaiac and three immunochemical faecal occult blood tests in screening for colorectal cancer. Eur J Cancer. 2012:48(16):2969-76.

26 Parente F, Vailati C, Boemo C, Bonoldi E, Ardizzoia A, llardo A, Tortorella F, Cereda D, Cremaschini M, Moretti R. Improved 5 -year survival of patients with immunochemical faecal blood test-screen-detected colorectal cancer versus non-screening cancers in northern Italy. Dig Liver Dis. 2015 Jan;47(1):68-72. 